\title{
Identification of potential gene targets in systemic vasculitis using DNA microarray analysis
}

\author{
YIWEN FENG ${ }^{1}$, MIAO ZHENG ${ }^{1}$, SHUJIE GAN ${ }^{1}$, LEI ZHANG ${ }^{1}$, ZHONG WAN $^{1}$, \\ YANPING ZHANG $^{1}$, QIN QIAN $^{1}$ and JINGDONG TANG ${ }^{1,2}$ \\ ${ }^{1}$ Vascular Surgery Department, Shanghai General Hospital of Nanjing Medical University, \\ Shanghai 200080; ${ }^{2}$ Department of Vascular Surgery, Shanghai Pudong Hospital, \\ Fudan University, Pudong Medical Center, Shanghai 201399, P.R. China
}

Received December 21, 2015; Accepted January 27, 2017

DOI: $10.3892 / \mathrm{mmr} .2017 .6455$

\begin{abstract}
The present study aimed to identify the involvement of critical genes in systemic vasculitis, to gain an improved understanding of the molecular circuity and to investigate novel potential gene targets for systemic vasculitis treatment. The dual-color cDNA microarray data of GSE16945, consisting of peripheral mononuclear blood cell specimens from 13 patients with systemic vasculitis and 16 healthy controls, was downloaded from the Gene Expression Omnibus database. Differentially expressed genes (DEGs) were screened in systemic vasculitis compared with controls using BRB ArrayTools, followed by the construction of a protein-protein interaction (PPI) network using the clusterProfiler package, and significant functional interaction (FI) module selection. Furthermore, transcriptional factors (TFs) among the identified DEGs were predicted and a transcriptional regulation network was constructed. A total of 173 up- and 93 downregulated genes were identified, which were mainly associated with immune response pathways. FBJ murine osteosarcoma viral oncogene homolog $(F O S)$, ubiquitin B $(U B B)$, signal transducer and activator of transcription 1 (STATI) and MX dynamin-like GTPase $1(M X 1)$ were identified as hub proteins in the PPI network. Furthermore, $U B B, F O S$, and $S T A T 1$ were hub proteins in the three identified FI modules, respectively. In total, nine TFs were predicted among the DEGs. Of the DEGs that were predicted to be TFs, STAT1, $v$-maf avian musculoaponeurotic fibrosarcoma oncogene homolog B $(M A F B)$ and tyrosine 3-monooxygenase/tryptophan 5-monooxygenase activation protein $\mathrm{Z}$ ( $Y W H A Z)$, which interacted with each other, were identified to regulate further DEGs as target genes. Various
\end{abstract}

Correspondence to: Dr Jingdong Tang, Department of Vascular Surgery, Shanghai Pudong Hospital, Fudan University, Pudong Medical Center, 2800 Gongwei Road, Pudong, Shanghai 201399, P.R. China

E-mail: drtangjingdong@126.com

Key words: systemic vasculitis, differentially expressed genes, protein-protein interaction network, transcriptional factors genes, including $F O S, U B B, M X 1$, STAT1, MAFB, and $Y W H A Z$ may be potential targets useful for the treatment of systemic vasculitis.

\section{Introduction}

Systemic vasculitis represents a heterogeneous group of disorders characterized by inflammation and necrosis in the blood vessel wall involving multiple organs (1). Clinical manifestations of systemic vasculitis are nonspecific and may vary from mild disorders to life-threatening multisystem conditions (2). Cases of vasculitis exhibit differences in pathology, type of inflammation, vessel and organ involvement, and demographics (3). Therefore, the diagnosis and treatment of systemic vasculitis is challenging, and improvements in therapy and the understanding of the etiopathogenesis are required. Although the etiology of systemic vasculitis is not fully understood, there are a combination of genetic, immunological and environmental factors that may be responsible for certain cases. Accumulating evidence has demonstrated that genetic factors contribute to the susceptibility to vasculitis (4). Anti-neutrophil cytoplasmic antibody-associated vasculitis (AAV) is a necrotizing group of disorders characterized by autoimmune inflammation that predominantly affects small to medium-sized vessels, which can lead to vessel occlusion and systemic organ damage (5). Ordonez et al (6) reported that CD45RC ${ }^{\text {low }} \mathrm{CD} 4 \mathrm{~T}$ cells were significantly increased in patients with AAV compared with healthy controls, which may contribute to the susceptibility to AAV. Additionally, Kobayashi et al (7) demonstrated that putative gene markers, particularly early growth response 1 and G0/G1 switch gene 2, may be useful for diagnosing vasculitis, and monocytes expressing these vasculitis-upregulated genes may be involved in the pathogenesis of vasculitis. However, the molecular mechanism underlying the development and progression of systemic vasculitis remains unclear. Continued investigation and identification of the genetic factors involved in the pathogenesis of systemic vasculitis is necessary.

Dual-color cDNA microarray data was used in the present study to identify differentially expressed genes (DEGs) in samples from patients with systemic vasculitis compared with healthy controls. Comprehensive bioinformatics were 
conducted to analyze the significant gene ontology (GO) terms and pathways that the DEGs were involved in. This was followed by the construction of a protein-protein interaction (PPI) network and significant functional interaction (FI) module selection. Furthermore, transcriptional factors (TFs) among the DEGs were predicted and, subsequently, a transcriptional regulation network was constructed. The current study aimed to identify the involvement of critical genes in systemic vasculitis, to obtain an improved understanding of the molecular circuity in systemic vasculitis and to investigate novel potential gene targets for systemic vasculitis treatment.

\section{Materials and methods}

Data source. The dual-color cDNA microarray data GSE16945 (8), was downloaded from the Gene Expression Omnibus database (http://www.ncbi.nlm.nih.gov/geo/) based on the platform of GPL4133 Agilent-014850 Whole Human Genome Microarray 4x44K G4112F (feature number version). This dual-color microarray dataset consisted of details of 13 microarray chips. Each chip included a cyanine (Cy) 3-labelled channel derived from mixed peripheral mononuclear blood cells (PMBCs) from 16 healthy volunteers as controls and a Cy5-labelled channel derived from PMBCs from a patient with systemic vasculitis. A total of 13 patients with vasculitis were included in the present study.

Data preprocessing and DEG screening. Raw data were imported into BRB ArrayTools (http://linus.nci.nih. gov/BRB-ArrayTools.html; version 4.3.1) developed by Simon et al (9). The preprocessing step included local background subtraction, averaging of intensities of duplicated probes and quantile normalization across multiple arrays. DEG screening was performed via the BRB ArrayTools package (9). Genes were excluded when $<50 \%$ of the expression data had $>1.5$-fold change in either direction from the median value of the gene. Genes were also excluded when the percentage of data filtered out or missing was $>50 \%$. The threshold for a DEG was $\mathrm{P}<0.05$ using multivariate permutation tests as previously described (10).

Functional and pathway enrichment analysis. The clusterProfiler package is implemented in $\mathrm{R}$, which is an open-source programming environment (11) and this package automates the process of biological-term enrichment analysis of gene clusters (12). In the present study, the clusterProfiler package version 3.4 (http://bioconductor.org/packages/release/ bioc/html/clusterProfiler.html) was applied to perform GO analysis (including cellular composition, molecular function and biological process terms) and Kyoto Encyclopedia of Genes and Genomes (KEGG) pathway enrichment analysis. False discovery rate (FDR) (13) was performed to adjust P-values using the Benjamini and Hochberg method (14). An FDR $<0.05$ was selected as the cutoff criterion.

PPI network construction. A PPI network was constructed in the present study using the Search Tool for the Retrieval of Interacting Genes database (15). Interacting pairs of DEGs (confidence score $>0.4$ ) (16) were selected for PPI network construction. Additionally, Cytoscape software version
2.8 (17) was used to provide interactive visualization for the PPI network. The node degrees in the PPI network were calculated with the igraph package version 0.5.3 (18) in R and the nodes with higher degrees were considered to be hub proteins.

FI network construction and modules selection. ReactomeFIViz (19), a Cytoscape plugin, integrates constructed human protein FI networks by combining curated and non-curated data sources, and the gene expression matrix, to calculate the Pearson coefficient of genes (20) in an FI network. In the FI network, the Pearson coefficient of each pair of genes were considered as edge weights. In addition, ReactomeFIViz further divides the FI network into modules using a popular algorithm, Markov Clustering (MCL) (21). In the current study, a gene FI network was constructed using ReactomeFIViz (19) and module division was performed. Furthermore, Gene Cluster with Literature Profiles (GenCLiP) software version 2.0, which was developed by Huang et al (22) to cluster gene lists by literature profiling and to construct gene co-occurrence networks associated with custom keywords, was used to analyze the biological behavior of genes identified in the selected modules.

Prediction of TFs and transcriptional regulation network construction. TRANSFAC is a database on TFs, which contains the genomic binding sites of TFs and DNA-binding profiles, and is an integrated system for gene expression regulation (23). TFs among the identified DEGs were screened for using data derived from TRANSFAC database in the present study. Candidate binding sites for the identified TFs in the promoter region were also identified through sequence matching of the position weight matrix (24) by using the MotifDb R package (25). The location of the TF binding site (TFBS) within the promoter region of each DEG was predicted using the position weight matrix algorithm, in which a minimum score for a match was set at $85 \%$. Subsequently, a transcriptional regulation network was constructed that included the identified TFs and other DEGs.

\section{Results}

Screening of DEGs. A total of 266 DEGs were identified in PMBCs from patients with systemic vasculitis compared with controls, including 173 up- and 93 downregulated genes. The results demonstrated that there were more upregulated genes than downregulated genes in patients with vasculitis compared with controls.

GO and pathway enrichment analysis. GO and pathway analysis demonstrated that up- and downregulated genes were associated with different GO terms and pathways. The top five GO terms in each category of the up- and downregulated genes are presented in Table I. Additionally, the top five KEGG pathways associated with the up- and downregulated genes are presented in Table II. The results of the present study demonstrated that upregulated DEGs were primarily involved in biological processes associated with defense response and response to stress or stimulus. However, downregulated DEGs were enriched in biological processes associated with immune responses, such as the innate immune response. 
Table I. Top five enriched BP, CC, and MF GO terms for up- and downregulated genes in patients with systemic vasculitis.

\begin{tabular}{|c|c|c|c|c|}
\hline GO ID & Description & P-value & $\begin{array}{c}\text { False } \\
\text { discovery rate }\end{array}$ & Count \\
\hline \multicolumn{5}{|l|}{ Upregulated } \\
\hline GO_BP 0006950 & Response to stress & $2.35 \mathrm{E}-12$ & $2.40 \mathrm{E}-09$ & 65 \\
\hline GO_BP 0002376 & Immune system process & $6.88 \mathrm{E}-12$ & $2.90 \mathrm{E}-09$ & 51 \\
\hline GO_BP 0006952 & Defense response & $8.52 \mathrm{E}-12$ & 2.90E-09 & 40 \\
\hline GO_BP 0006954 & Inflammatory response & $3.24 \mathrm{E}-10$ & 8.28E-08 & 23 \\
\hline GO_BP 0050896 & Response to stimulus & $9.57 \mathrm{E}-10$ & $1.96 \mathrm{E}-07$ & 101 \\
\hline GO_CC 0031982 & Vesicle & $7.96 \mathrm{E}-09$ & $6.77 \mathrm{E}-07$ & 58 \\
\hline GO_CC 0044421 & extracellular region part & $1.32 \mathrm{E}-08$ & $6.77 \mathrm{E}-07$ & 59 \\
\hline GO_CC 0005833 & Hemoglobin complex & $1.51 \mathrm{E}-08$ & $6.77 \mathrm{E}-07$ & 5 \\
\hline GO_CC 0005615 & Extracellular space & $3.96 \mathrm{E}-08$ & $1.33 \mathrm{E}-06$ & 30 \\
\hline GO_CC 0031988 & Membrane-bounded vesicle & $1.69 \mathrm{E}-07$ & $3.80 \mathrm{E}-06$ & 54 \\
\hline GO_MF 0003674 & Molecular function & $3.42 \mathrm{E}-10$ & 4.82E-08 & 146 \\
\hline GO_MF 0005515 & Protein binding & $3.00 \mathrm{E}-08$ & $1.33 \mathrm{E}-06$ & 100 \\
\hline GO_MF 0005488 & binding & $3.11 \mathrm{E}-08$ & $1.33 \mathrm{E}-06$ & 129 \\
\hline GO_MF 0005344 & Oxygen transporter activity & $3.76 \mathrm{E}-08$ & $1.33 \mathrm{E}-06$ & 5 \\
\hline GO_MF 0005506 & Iron ion binding & $8.50 \mathrm{E}-06$ & 0.000212228 & 9 \\
\hline \multicolumn{5}{|l|}{ Downregulated } \\
\hline GO_BP 0006952 & Defense response & $2.30 \mathrm{E}-16$ & $8.58 \mathrm{E}-14$ & 34 \\
\hline GO_BP 0006955 & Immune response & $2.46 \mathrm{E}-16$ & $8.58 \mathrm{E}-14$ & 33 \\
\hline GO_BP 0045087 & Innate immune response & $4.35 \mathrm{E}-14$ & $1.01 \mathrm{E}-11$ & 25 \\
\hline GO_BP 0002376 & Immune system process & $7.59 \mathrm{E}-14$ & $1.32 \mathrm{E}-11$ & 38 \\
\hline GO_BP 0051607 & Defense response to virus & $4.92 \mathrm{E}-13$ & $6.86 \mathrm{E}-11$ & 14 \\
\hline GO_CC 0044444 & Cytoplasmic part & $1.86 \mathrm{E}-06$ & 0.000161279 & 55 \\
\hline GO_CC 0005737 & Cytoplasm & $2.56 \mathrm{E}-06$ & 0.000161279 & 66 \\
\hline GO_CC 0042611 & MHC protein complex & $7.12 \mathrm{E}-06$ & 0.000298999 & 4 \\
\hline GO_CC 0071556 & $\begin{array}{l}\text { Integral component of lumenal } \\
\text { side of endoplasmic reticulum } \\
\text { membrane }\end{array}$ & 0.000294636 & 0.005946701 & 3 \\
\hline GO_CC 0098553 & $\begin{array}{l}\text { Lumenal side of endoplasmic } \\
\text { reticulum membrane }\end{array}$ & 0.000294636 & 0.005946701 & 3 \\
\hline GO_MF 0003674 & Molecular function & $3.66 \mathrm{E}-06$ & 0.00019454 & 84 \\
\hline GO_MF 0003823 & Antigen binding & $4.42 \mathrm{E}-06$ & 0.00019454 & 6 \\
\hline GO_MF 0005515 & Protein binding & $1.59 \mathrm{E}-05$ & 0.000466985 & 58 \\
\hline GO_MF 0003924 & GTPase activity & $6.22 \mathrm{E}-05$ & 0.001368961 & 7 \\
\hline GO_MF 0005525 & GTP binding & 0.000282526 & 0.003941692 & 8 \\
\hline
\end{tabular}

GO, gene ontology; BP, biological process; CC, cellular composition; MF, molecular function. Count, the number of DEGs that this specific GO-ID term was associated with.

PPI network construction and analysis. The PPI network was constructed and is presented in Fig. 1. The PPI network included 163 nodes and 449 interactions, involving 107 upand 56 downregulated genes. Following the calculation of node degrees, the present study identified that the top five DEGs in the PPI network, with higher node degrees, were FBJ murine osteosarcoma viral oncogene homolog (FOS; degree, 23), ubiquitin B (UBB; degree, 22), ISG15 ubiquitin-like modifier (degree, 22), signal transducer and activator of transcription 1 (STAT1; degree, 21) and MX dynamin-like GTPase 1 (MX1; degree, 17). Furthermore, a total of nine
TFs that were involved in the PPI network were predicted, including cold shock domain protein A, Fas cell surface death receptor, high mobility group box 2 , interferon regulatory factor 5, $v$-maf avian musculoaponeurotic fibrosarcoma oncogene homolog B $(M A F B)$, myocyte enhancer factor $2 \mathrm{D}$ (MEF 2D), POU class 2 associating factor 1, STAT1, tyrosine 3-monooxygenase/tryptophan 5-monooxygenase activation protein $\mathrm{Z}(Y W H A Z)$.

Module selection in the FI network and function analysis of DEGs in the modules. The FI network of DEGs was 
Table II. Enriched KEGG pathways for up- and downregulated genes in patients with systemic vasculitis.

\begin{tabular}{|c|c|c|c|c|}
\hline KEGG ID & Description & P-value & $\begin{array}{c}\text { False } \\
\text { discovery rate }\end{array}$ & Count \\
\hline $\begin{array}{l}\text { Upregulated } \\
\text { hsa05130 }\end{array}$ & $\begin{array}{l}\text { Pathogenic Escherichia } \\
\text { coli infection }\end{array}$ & 0.000614223 & 0.013512902 & 5 \\
\hline hsa03010 & Ribosome & 0.004807379 & 0.035254116 & 5 \\
\hline hsa05323 & Rheumatoid arthritis & 0.004807379 & 0.035254116 & 5 \\
\hline hsa05143 & African trypanosomiasis & 0.008320256 & 0.038297864 & 3 \\
\hline hsa05146 & Amoebiasis & 0.008704060 & 0.038297864 & 5 \\
\hline \multicolumn{5}{|c|}{ Downregulated } \\
\hline hsa05330 & Allograft rejection & 0.000139043 & 0.001547821 & 4 \\
\hline hsa05332 & Graft-versus-host disease & 0.000204470 & 0.001547821 & 4 \\
\hline hsa04940 & Type I diabetes mellitus & 0.000244393 & 0.001547821 & 4 \\
\hline hsa05320 & Autoimmune thyroid disease & 0.000495821 & 0.002355147 & 4 \\
\hline hsa04145 & Phagosome & 0.000652198 & 0.002478353 & 6 \\
\hline
\end{tabular}

Count, the number of DEGs that the specific KEGG-ID was associated with.

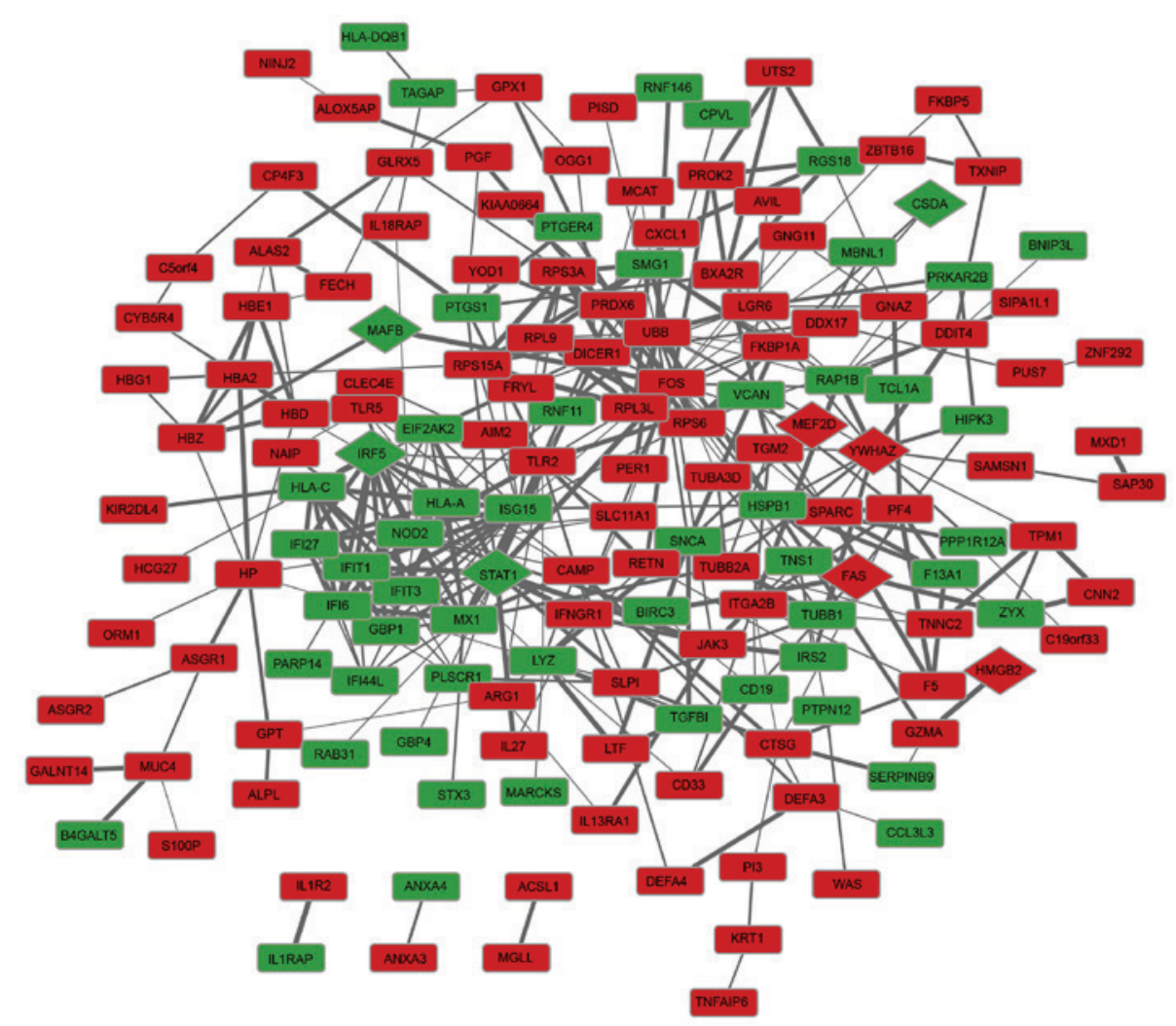

Figure 1. Protein-protein interaction network of differentially expressed genes in patients with systemic vasculitis compared with controls. Red nodes indicate upregulated genes. Green nodes indicate downregulated genes. Diamond-shaped nodes indicate transcription factors.

constructed using the ReactomeFIViz plugin. Furthermore, three significant modules were identified using the MCL algorithm (Fig. 2). UBB, FOS and STAT1 were identified as hub proteins for one of the three identified modules. In addition, these three DEGs interacted with each other, and FOS and STAT1 were identified as TFs following literature profiling. As presented in Fig. 2, there were seven, nine and seven DEGs enriched in modules 1, 2 and 3, respectively. Additionally, according to the clustered gene lists by literature profiling, it was identified that the upregulated DEG, $U B B$, which was the hub protein in module 1 , was associated with cell death and signal transduction (Fig. 3). While the hub protein in module 2, FOS (upregulated), as an important TF, was associated with inflammatory response, signal transduction and cell 


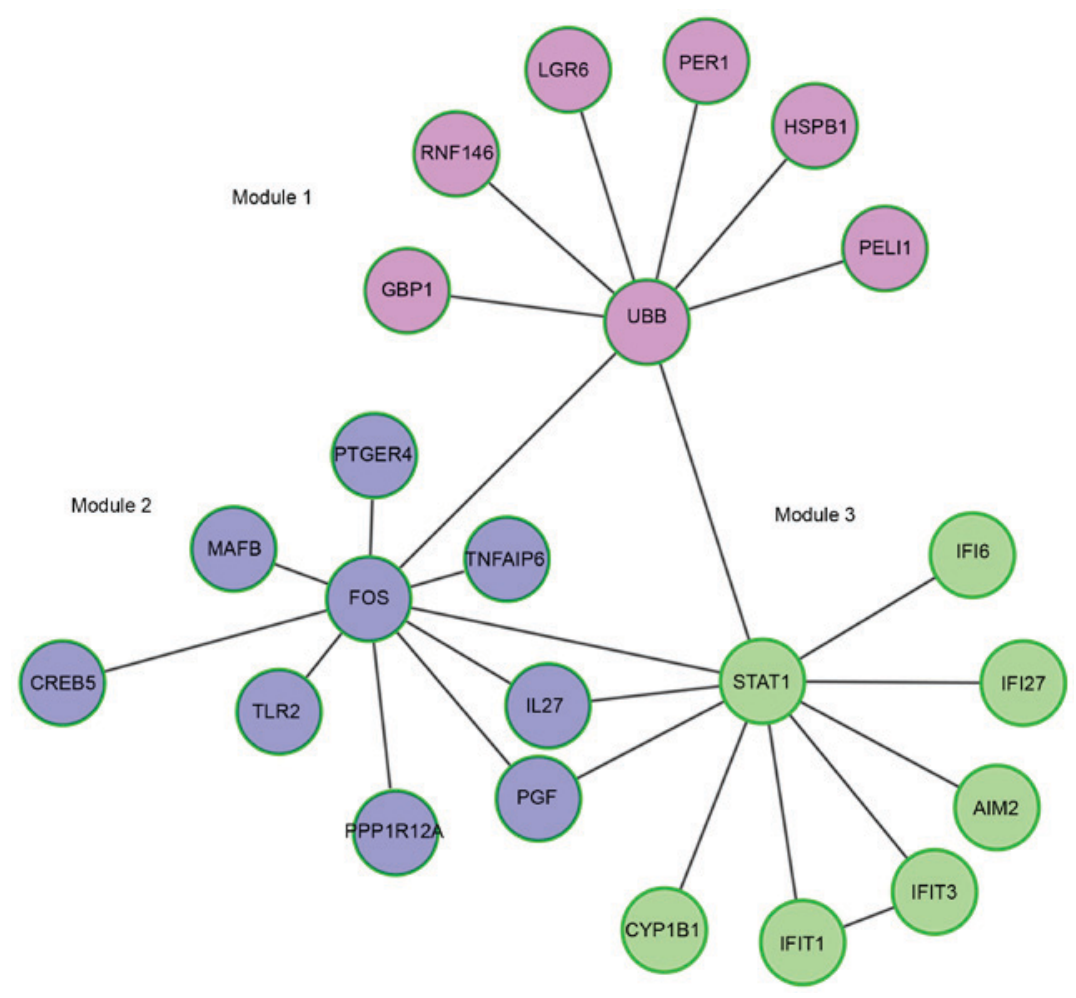

Figure 2. Functional interaction modules of differentially expressed genes in patients with systemic vasculitis compared with controls. GBP1, guanylate binding protein 1; RNF146, ring finger protein 146; LGR6, leucine rich repeat containing G protein-coupled receptor 6; PER1, period circadian clock 1; HSPB1, heat shock protein family B (small) member 1; PELI1, pellino E3 ubiquitin protein ligase 1; UBB, ubiquitin B; PTGER4, prostaglandin E receptor 4; MAFB, $v$-maf avian musculoaponeurotic fibrosarcoma oncogene homolog B; CREB5, cAMP responsive element binding protein 5; TNFAIP6, TNF $\alpha$ induced protein 6; IL27, interleukin 27; PGF, placental growth factor; PPP1R12A, protein phosphatase 1 regulatory subunit 12A; TLR2, Toll-like receptor 2; FOS, FBJ murine osteosarcoma viral oncogene homolog; IFI, interferon $\alpha$ inducible protein; AIM2, absent in melanoma 2; IFIT, interferon induced protein with tetratricopeptide repeats; CYP1B1, cytochrome P450 family 1 subfamily B member 1; STAT1, signal transducer and activator of transcription 1.
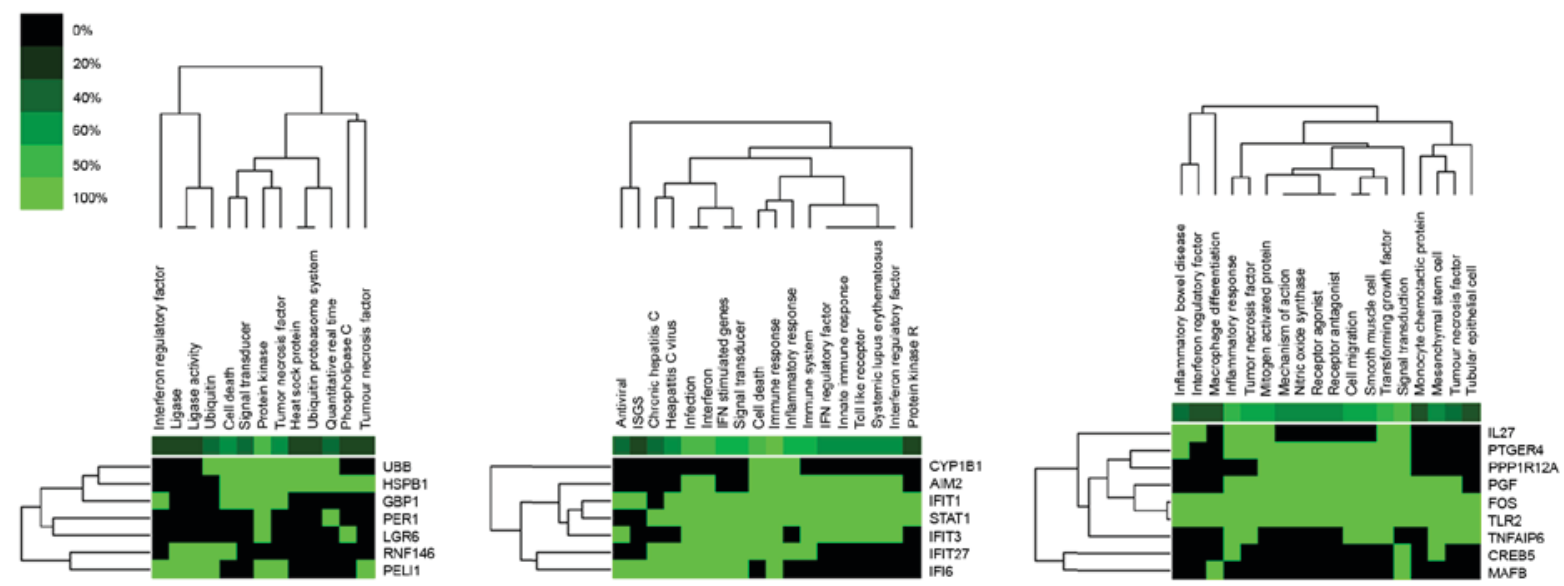

Figure 3. Gene function in 3 functional interaction modules identified with the Markov Clustering algorithm. Green indicates corresponding gene-term association positively reported. Black represents corresponding gene-term association not yet reported. UBB, ubiquitin B; HSPB1, heat shock protein family B (small) member 1; GBP1, guanylate binding protein 1; PER1, period circadian clock 1; LGR6, leucine rich repeat containing G protein-coupled receptor 6; RNF146, ring finger protein 146; PELI1, pellino E3 ubiquitin protein ligase 1; CYP1B1, cytochrome P450 family 1 subfamily B member 1; AIM2, absent in melanoma 2; IFIT, interferon induced protein with tetratricopeptide repeats; STAT1, signal transducer and activator of transcription 1; IFI6, interferon $\alpha$ inducible protein 6; IL27, interleukin 27; PTGER4, prostaglandin E receptor 4; PPP1R12A, protein phosphatase 1 regulatory subunit 12A; PGF, placental growth factor; FOS, FBJ murine osteosarcoma viral oncogene homolog; TLR2, Toll-like receptor 2; TNFAIP6, TNF $\alpha$ induced protein 6; CREB5, cAMP responsive element binding protein 5; MAFB, $v$-maf avian musculoaponeurotic fibrosarcoma oncogene homolog B.

migration. Downregulated STATl, which was the hub protein in module 3, was identified to be a TF that was associated with the immune response, inflammatory response, cell death and Toll-like receptors (TLRs).
Construction and analysis of the transcriptional regulation network. As mentioned above, a total of nine DEGs were predicted to be TFs based on data derived from the TRANSFAC database. The present study subsequently predicted all of the 


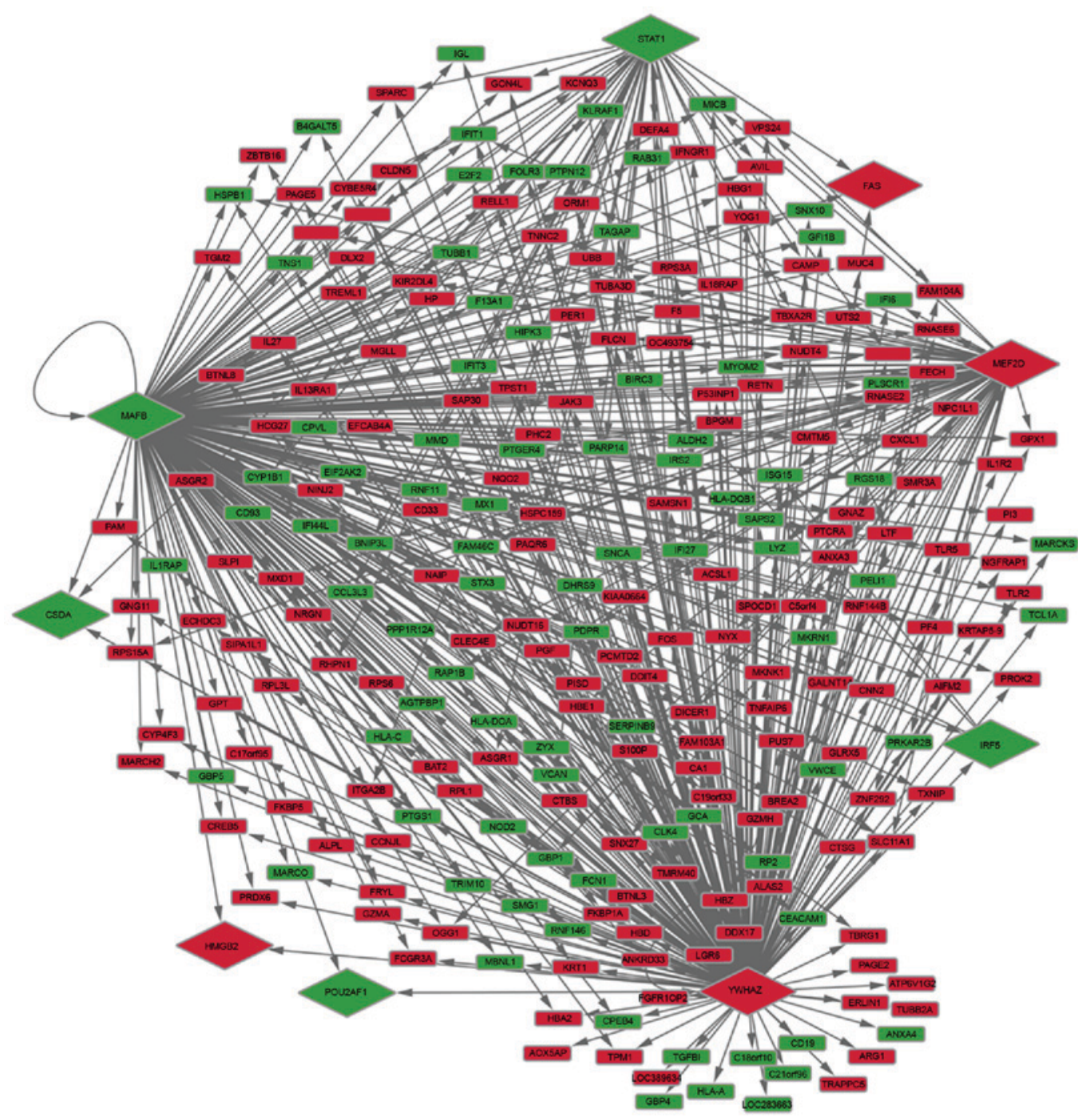

Figure 4. Transcriptional regulation network. Red nodes indicate upregulated genes. Green nodes indicate downregulated genes. Diamond-shaped nodes indicate transcription factors.

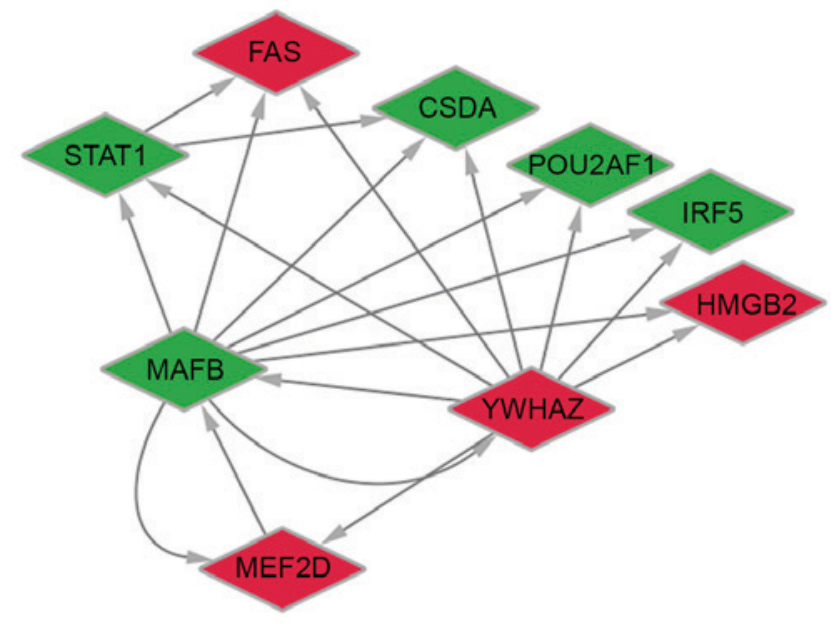

Figure 5. Interactions among the predicted transcription factors. Red nodes indicate upregulated genes. Green nodes indicate downregulated genes. STAT1, signal transducer and activator of transcription 1; FAS, Fas cell surface death receptor; CSDA, cold shock domain protein A; POU2AF1, POU class 2 associating factor 1; IRF5, interferon regulatory factor 5; HMGB2, high mobility group box 2; YWHAZ, tyrosine 3-monooxygenase/tryptophan 5-monooxygenase activation protein Z; MEF2D, myocyte enhancer factor 2D; MAFB, $v$-maf avian musculoaponeurotic fibrosarcoma oncogene homolog B potential target genes, which were also identified as DEGs, of these nine TFs. The results demonstrated that five TFs potentially regulated 257 DEGs (167 up- and 90 downregulated genes). In particular, it was identified that STAT1, MEF2D, MAFB and $Y W H A Z$ potentially regulated further DEGs (Fig. 4). TFBS were also identified in pairs of TFs. The interactions among the nine identified TFs are presented in Fig. 5. Furthermore, genes that were coregulated by STAT1, MEF $2 D, M A F B$, and $Y W H A Z$ were analyzed. The results indicated that TFs $S T A T 1$ and $M A F B$ may coregulate 39 target genes, while $M E F 2 D$ and $Y W H A Z$ may coregulate 70 target genes. These target genes were enriched in biological processes associated with response to environmental stimulus and immune response (Fig. 6).

\section{Discussion}

Gene expression profiling using DNA microarrays is a tool for investigating systemic vasculitis at a molecular level $(4,7)$. In the current study, a total of 173 up- and 93 downregulated genes were identified in PMBCs from patients with systemic vasculitis compared with controls. GO and pathway enrichment analysis demonstrated that DEGs were primarily 


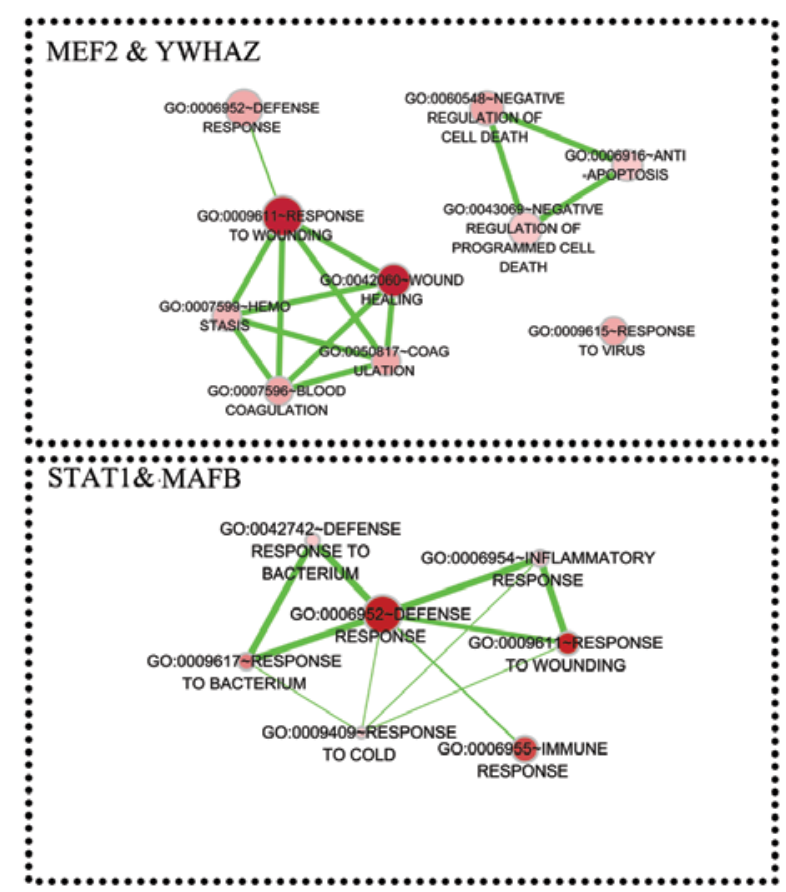

Figure 6. Enriched GO-BP terms of genes that were co-regulated by STAT1 and $M A F B$, and GO-BP terms of genes that were co-regulated by $M E F 2$ and $Y W H A Z$. GO, gene ontology; BP, biological process; MEF2, myocyte enhancer factor 2D; YWHAZ, tyrosine 3-monooxygenase/tryptophan 5-monooxygenase activation protein Z; STAT1, signal transducer and activator of transcription 1; MAFB, $v$-maf avian musculoaponeurotic fibrosarcoma oncogene homolog B.

associated with immune response. FOS, UBB, STAT1 and $M X 1$ were identified as hub proteins in the PPI network. Furthermore, $U B B, F O S$ and $S T A T 1$ were hub proteins in one of the three identified FI modules. A total of nine TFs were predicted among the identified DEGs. Of those nine TFs, STAT1, MAFB and $Y W H A Z$, which exhibited interactions among each other, were indicated to regulate further DEGs as target genes in the regulation network. The target genes of the TFs were primarily associated with response to environmental stimulus and immune response.

The FOS gene family encodes leucine zipper proteins, which dimerize with proteins of the JUN family, forming the TF complex activator protein-1 (AP-1) to regulate gene expression (5). Previous studies have demonstrated that the signaling of TLRs, which results in cytokine production, is integrated by adapter molecules that activate AP-1 (FOS/JUN) (26-28). In addition, Tadema et al (29) demonstrated that monocytes and natural killer cells exhibited increased TLR expression in AAV. Consistent with the previous study, the results of the current study revealed that FOS was upregulated in PMBCs from patients with systemic vasculitis compared with controls, and FOS was a hub protein in the PPI network and also in the FI module 2. In this context, we hypothesized that FOS may have a crucial role in the TLR signaling involved in the inflammatory response in systemic vasculitis, and, thus, FOS may be a potential gene target for vasculitis treatment.

$U B B$ encodes ubiquitin, which is one of the most conserved proteins and has a major role in targeting cellular proteins for degradation by the $26 \mathrm{~S}$ proteasome (30). It is apparent that ubiquitination of various components of the Notch signaling pathway functions in shaping and orchestrating the Notch signaling pathway (31). Additionally, Piggott et al (32) revealed that blocking the Notch pathway inhibited vascular inflammation in large-vessel vasculitis and modulating the Notch signaling cascade may be a promising novel method for immunosuppressive therapy of large-vessel vasculitis. Furthermore, the present study identified that $U B B$ was upregulated in PMBCs from patients with systemic vasculitis compared with controls, and $U B B$ was a hub protein in the PPI network and in the identified FI module 1. Combined, these results indicate that upregulated $U B B$ may function in the progression of systemic vasculitis via participation in the Notch signaling pathway. Therefore, $U B B$ may be a potential therapeutic target for systemic vasculitis, which requires further investigation.

The results of the present study also identified that $M X 1$ was another hub protein in the PPI network. $M X 1$ encodes a GTP-metabolizing protein, which is induced by type I and type II interferons and participates in the cellular antiviral response by antagonizing the replication process of several viruses (33). Evidence has demonstrated that the most common vasculitic syndrome associated with hepatitis $\mathrm{C}$ virus ( $\mathrm{HCV}$ ) infection is an immune complex-mediated type of systemic vasculitis, preferentially involving the small vessels (34). MX1 may be associated with interferon signaling and have an essential role in the cellular antiviral response that is involved in systemic vasculitis, which requires further investigation.

Furthermore, the present study demonstrated that the predicted TFs, STAT1, MAFB, and $Y W H A Z$, which interacted with each other, regulated further DEGs as target genes in the regulation network, indicating the importance of them. The protein encoded by STAT1 is a member of the STAT protein family, which are phosphorylated by receptor associated kinases, and subsequently act as transcriptional activators. Previous studies have demonstrated that STAT acts as a signal transducer and transcriptional activator, and mediates cellular responses to interferons, other cytokines and growth factors (35-37). Lin et al (38) demonstrated that HCV suppressed interferon signaling by degrading STAT1. Chan et al (39) reported that type I interferons, when used to treat resistant Churg-Strauss syndrome, a type of systemic vasculitis, led to complete remission in $25 \%$ of a small case series. In a previous study, it was observed that $M A F B$ suppressed acute inflammatory responses in lipopolysaccharide-stimulated lung injury (40). Additionally, it was demonstrated that $M A F B$ modulated the efficiency of interferon production. On the other hand, $Y W H A Z$ belongs to the 14-3-3 family of proteins, which mediate signal transduction and gene regulation events through binding to phosphoserine-containing proteins (41). Nishimura et al (41) demonstrated that overexpression of $Y W H A Z$ may inhibit cell apoptosis in breast cancer cell lines. In addition, the activation of STAT1 was demonstrated to induce apoptosis (42). Furthermore, Jamin et al (43) revealed that apoptosis of endothelial cells was induced by the binding of anti-endothelial cell antibodies to heat shock protein family D (Hsp60) member 1 in vasculitis-associated systemic autoimmune diseases. We hypothesized that STAT1 may be involved in interferon signaling transduction via interaction 
with $M A F B$, and STAT1 may participate in cell apoptosis through interaction with $Y W H A Z$ in systemic vasculitis.

In conclusion, the critical genes involved in systemic vasculitis have been investigated based on the microarray data used in this study. FOS may function in TLR signaling that is involved in the inflammatory response and $U B B$ may function in the progression of systemic vasculitis via participation in the Notch signaling pathway. In addition, $M X 1$ may be associated with interferon signaling and have an essential role in the cellular antiviral response in systemic vasculitis. Furthermore, STAT1 may be involved in interferon signaling transduction via interaction with $M A F B$, and STAT1 may participate in cell apoptosis through interaction with $Y W H A Z$ in systemic vasculitis. Further experiments and investigation with larger sample sizes are required to verify these results. Insights into promising gene targets should lead to novel and effective strategies, and improved targeted therapy for systemic vasculitis.

\section{Acknowledgements}

This study was supported by the Shanghai Science and Technology Commission fund (grant no. 15495810202), Medical Engineering Cross Research fund (Shanghai Jiaotong University; grant no. YG2013MS08) and Medical Engineering Cross Research fund (Shanghai Jiaotong Universityy; grant no. YG2014QN09).

\section{References}

1. de Souza AWS: Autoantibodies in systemic vasculitis. Front Immunol 6: 184, 2015.

2. Stagnaro C, Cioffi E, Talarico R and Della Rossa A: Systemic vasculitides: A critical digest of the most recent literature. Clin Exp Rheumatol 33 (2 Suppl 89): S145-S154, 2015.

3. Jennette JC, Falk RJ, Bacon PA, Basu N, Cid MC, Ferrario F, Flores-Suarez LF, Gross WL, Guillevin L, Hagen EC, et al: 2012 revised international chapel hill consensus conference nomenclature of vasculitides. Arthritis Rheumatism 65: 1-11, 2013.

4. Yang JJ, Preston GA, Alcorta DA, Waga I, Munger WE, Hogan SL, Sekura SB, Phillips BD, Thomas RP, Jennette JC and Falk RJ: Expression profile of leukocyte genes activated by anti-neutrophil cytoplasmic autoantibodies (ANCA). Kidney Int 62: 1638-1649, 2002.

5. Wagner EF: Bone development and inflammatory disease is regulated by AP-1 (Fos/Jun). Annals Rheum Dis 69: i86-i88, 2010.

6. Ordonez L, Bernard I, L'Faqihi-Olive FE, Tervaert JW, Damoiseaux J and Saoudi A: CD45RC isoform expression identifies functionally distinct $T$ cell subsets differentially distributed between healthy individuals and AAV patients. PLoS One 4: e5287, 2009.

7. Kobayashi S, Ito A, Okuzaki D, Onda H, Yabuta N, Nagamori I, Suzuki K, Hashimoto H and Nojima H: Expression profiling of PBMC-based diagnostic gene markers isolated from vasculitis patients. DNA Res 15: 253-265, 2008

8. Okuzaki D, Fukushima T, Tougan T, Ishii T, Kobayashi S, Yoshizaki K, Akita T and Nojima H: Genopal ${ }^{\mathrm{TM}}$ : A novel hollow fibre array for focused microarray analysis. DNA Res 17: 369-379, 2010.

9. Simon R, Lam A, Li MC, Ngan M, Menenzes S and Zhao Y: Analysis of gene expression data using BRB-array tools. Cancer Inform 3: 11-17, 2007.

10. Zhao Y and Simon R: BRB-ArrayTools Data Archive for human cancer gene expression: A unique and efficient data sharing resource. Cancer Inform 6: 9-15, 2008.

11. Ihaka R and Gentleman R: R: A language for data analysis and graphics. J Computational and graphical statistics 5: 299-314, 1996.

12. Yu G, Wang LG, Han Y and He QY: ClusterProfiler: An R package for comparing biological themes among gene clusters. Omics 16: 284-287, 2012.
13. Reiner-Benaim A: FDR control by the $\mathrm{BH}$ procedure for Two-Sided Correlated Tests with implications to gene expression Data Analysis. Biom J 49: 107-126, 2007.

14. Benjamini Y and Hochberg Y: Controlling the false discovery rate: A practical and powerful approach to multiple testing. J Royal Statistical Soc 57: 289-300, 1995.

15. Franceschini A, Szklarczyk D, Frankild S, Kuhn M, Simonovic M, Roth A, Lin J, Minguez P, Bork P, von Mering C and Jensen LJ: STRING v9.1: Protein-protein interaction networks, with increased coverage and integration. Nucleic Acids Res 41: D808-D815, 2013.

16. Yu J and Finley RL: Combining multiple positive training sets to generate confidence scores for protein-protein interactions. Bioinformatics 25: 105-111, 2009.

17. Shannon P, Markiel A, Ozier O, Baliga NS, Wang JT, Ramage D, Amin N, Schwikowski B and Ideker T: Cytoscape: A software environment for integrated models of biomolecular interaction networks. Genome Res 13: 2498-2504, 2003.

18. Csardi $G$ and Nepusz T: The igraph software package for complex network research. Inter Journal Complex Systems 1695: $1-9,2006$

19. Wu G, Dawson E, Duong A, Haw R and Stein L: ReactomeFIViz: A Cytoscape app for pathway and network-based data analysis. F1000Res 3: 146, 2014.

20. Tan PK, Downey TJ, Spitznagel EL Jr, Xu P, Fu D, Dimitrov DS, Lempicki RA, Raaka BM and Cam MC: Evaluation of gene expression measurements from commercial microarray platforms. Nucleic Acids Res 31: 5676-5684, 2003.

21. Van Dongen SM: Graph clustering by flow simulation. 2001.

22. Huang ZX, Tian HY, Hu ZF, Zhou YB, Zhao J and Yao KT: GenCLiP: A software program for clustering gene lists by literature profiling and constructing gene co-occurrence networks related to custom keywords. BMC bioinformatics 9: 308, 2008.

23. Matys V, Fricke E, Geffers R, Gössling E, Haubrock M, Hehl R, Hornischer K, Karas D, Kel AE, Kel-Margoulis OV, et al: TRANSFAC: Transcriptional regulation, from patterns to profiles. Nucleic Acids Res 31: 374-378, 2003.

24. Song KH, Kim YH and Kim BY: Sho-saiko-to, a traditional herbal medicine, regulates gene expression and biological function by way of microRNAs in primary mouse hepatocytes. BMC Complement Altern Med 14: 14, 2014.

25. Nicolle R, RadvanyiF and Elati M:CoRegNet: Reconstruction and integrated analysis of co-regulatory networks. Bioinformatics 31: 3066-3068, 2015.

26. Kawai T, Sato S, Ishii KJ, Coban C, Hemmi H, Yamamoto M, Terai K, Matsuda M, Inoue J, Uematsu S, et al: Interferon-alpha induction through Toll-like receptors involves a direct interaction of IRF7 with MyD88 and TRAF6. Nat Immunol 5: 1061-1068, 2004.

27. Dillon S, Agrawal A, Van Dyke T, Landreth G, McCauley L, Koh A, Maliszewski C, Akira S and Pulendran B: A Toll-like receptor 2 ligand stimulates Th2 responses in vivo, via induction of extracellular signal-regulated kinase mitogen-activated protein kinase and c-Fos in dendritic cells. J Immunol 172: 4733-4743, 2004.

28. Wagner EF and Eferl R: Fos/AP-1 proteins in bone and the immune system. Immunol Rev 208: 126-140, 2005.

29. Tadema H, Abdulahad WH, Stegeman CA, Kallenberg CG and Heeringa P: Increased expression of Toll-like receptors by monocytes and natural killer cells in ANCA-associated vasculitis. PLoS One 6: e24315, 2011.

30. Oh C, Park S, Lee EK and Yoo YJ: Downregulation of ubiquitin level via knockdown of polyubiquitin gene Ubb as potential cancer therapeutic intervention. Sci Rep 3: 2623, 2013.

31. Le Bras S, Loyer N and Le Borgne R: The multiple facets of ubiquitination in the regulation of notch signaling pathway. Traffic 12: 149-161, 2011.

32. Piggott K, Deng J, Warrington K, Younge B, Kubo JT, Desai M, Goronzy JJ and Weyand CM: Blocking the NOTCH pathway inhibits vascular inflammation in large-vessel vasculitisclinical perspective. Circulation 123: 309-318, 2011.

33. Verhelst J, Parthoens E, Schepens B, Fiers W and Saelens X: Interferon-inducible protein Mx1 inhibits influenza virus by interfering with functional viral ribonucleoprotein complex assembly. J Virol 86: 13445-13455, 2012.

34. Vassilopoulos D and Calabrese LH: Hepatitis C virus infection and vasculitis: Implications of antiviral and immunosuppressive therapies. Arthritis Rheum 46: 585-597, 2002.

35. Ramana CV, Chatterjee-Kishore M, Nguyen H and Stark GR: Complex roles of Stat in regulating gene expression. Oncogene 19: 2619-2627, 2000. 
36. Kawazoe Y, Naka T, Fujimoto M, Kohzaki H, Morita Y, Narazaki M, Okumura K, Saitoh H, Nakagawa R, Uchiyama Y, et al: Signal transducer and activator of transcription (STAT)-induced STAT inhibitor 1 (SSI-1)/suppressor of cytokine signaling 1 (SOCS1) inhibits insulin signal transduction pathway through modulating insulin receptor substrate 1 (IRS-1) phosphorylation. J Exp Med 193: 263-270, 2001.

37. Klampfer L: Signal transducers and activators of transcription (STATs): Novel targets of chemopreventive and chemotherapeutic drugs. Curr Cancer Drug Targets 6: 107-121, 2006.

38. Lin W, Choe WH, Hiasa Y, Kamegaya Y, Blackard JT, Schmidt EV and Chung RT: Hepatitis C virus expression suppresses interferon signaling by degrading STAT1. Gastroenterology 128 : 1034-1041, 2005.

39. Chan AT, Flossmann O, Mukhtyar C, Jayne D and Luqmani RA The role of biologic therapies in the management of systemic vasculitis. Autoimmu Rev 5: 273-278, 2006.
40. Aida Y, Sato-Nishiwaki M, Abe S, Kishi H, Nunomiya K, Yamauchi K, lnoue S and Shibata Y: MafB suppresses acute inflammatory responses in lipopolysaccharide-stimulated lung injury in mice. Am J Respir Crit Care Med 185: A1340, 2012.

41. Nishimura Y, Komatsu S, Ichikawa D, Nagata H, Hirajima S, Takeshita H, Kawaguchi T, Arita T, Konishi H, Kashimoto K, et al: Overexpression of YWHAZ relates to tumor cell proliferation and malignant outcome of gastric carcinoma. Br J Cancer 108: 1324-1331, 2013.

42. Kaganoi J, Watanabe G, Okabe M, Nagatani S, Kawabe A, Shimada Y, Imamura M and Sakai Y: STAT1 activation-induced apoptosis of esophageal squamous cell carcinoma cells in vivo. Ann Surg Oncol 14: 1405-1415, 2007.

43. Jamin C, Duguuguabe G, Okabe M, et al: STAT1 activation-induced apoptosis of esophageal squamous cell carcinoma cells in vivo. Annals of Surgical Oncology 14: 1405-1415, 2007. 